\title{
Closed-Form Blind and Semi-Blind Estimation of Linear Receivers for Space-Time Coding
}

\author{
A. Lee Swindlehurst \\ Dept. of Electrical \& Computer Engineering \\ Brigham Young University \\ Provo, UT 84602
}

\begin{abstract}
This paper exploits a special class of space-time codes in which linearly transformed versions of a given data sequence are transmitted from multiple antennas. Several recently proposed codes, including space-time block codes, are members of this class. The redundancy introduced by the transformations imposes structure on the received data that under certain conditions can be exploited for direct blind (and semi-blind) estimation of a linear zero-forcing receiver that recovers the original data sequence. If the transmitted symbols are constant modulus, the space-time code structure can be exploited by the Analytic Constant Modulus (ACM) algorithm to simplify the separation of multiple co-channel users. If each user employs a different code or only one user is present, the ACM joint diagonalization step can be eliminated even though multiple constant modulus data streams are received.
\end{abstract}

\section{INTRODUCTION}

The throughput and diversity gains of systems employing multiple antennas on both ends of a wireless communication link can often only be exploited once the multiple-input multiple-output (MIMO) channel separating the transmitter and receiver has been identified. While training data can be used to estimate the channel, this approach consumes precious bandwidth and reduces throughput. The large body of previous research on blind multiuser, multichannel estimation and equalization is applicable to the MIMO problem, since the data broadcast from different transmit antennas can be thought of as data from different users. However, only recently have techniques appeared that exploit the structure built into space-time encoded signals. Many of these techniques have focused on the special structure of the so-called space-time block codes (STBC) described in [1], [2], [3], or generalizations thereof. Examples of methods that exploit STBC for blind and semiblind channel estimation include [4], [5], [6], [7]. Subspacebased approaches have been presented for modulation-induced coding in [8], [9], [10] and for circulant codes in [11].

In this paper, a different approach is presented in which a linear receiver is blindly (or semi-blindly) estimated directly from the data by exploiting the structure of certain space-time codes. The basic assumption underlying the proposed algorithm is that the symbols broadcast from the different transmit antennas are linear transformations of one another. This framework is general enough to include the STBCs of [1], [2], [3] as well as the specific codes used in [8], [9], [12], [13], [10], [11] as special cases. In the noiseless case, this property will cause the zero-forcing receivers for each transmit antenna to be related via linear transformations that, under certain conditions, can be determined directly from the data without knowledge of the channel. This fact is used to derive a deterministic, subspace-based estimator of a single weight vector that recovers the unique information-bearing transmitted sequence. The presence of any known training symbols in the data can also be easily exploited to improve the quality of the receiver estimate.

While the details of the algorithm are presented for the single user flat fading case, extensions to the multiple user case are discussed, along with modifications of the algorithm required when there are more transmit than receive antennas or the channel is rank deficient. When the symbols are constant modulus, the linear space-time code structure discussed above can be combined with that imposed by the Analytic Constant Modulus (ACM) algorithm [14] to improve performance. In the single user case, or when multiple users employ different space-time codes, the ACM joint diagonalization step can be eliminated in estimating the linear receiver, which considerably simplifies the algorithm.

\section{Data Model}

For the moment, assume a single-user transmit array with $K>1$ elements, a receive array with $M>1$ elements, and a flat-fading channel. If the receive array is sampled once per symbol over $N$ consecutive symbol periods, the following model results:

$$
\hat{\mathbf{X}}=\mathbf{H} \mathbf{S}_{K}+\mathbf{N}
$$

where $\hat{\mathbf{X}}$ is an $M \times N$ matrix of received data, $\mathbf{H}$ is the $M \times K$ channel matrix, $\mathbf{N}$ is additive noise and interference, and $\mathbf{S}_{K}$ is an $K \times N$ matrix containing the transmitted symbols. The subscript on $\mathbf{S}$ is used to explicitly indicate the number of rows in the matrix. The $(\hat{.})$ symbol is used to differentiate $\hat{\mathbf{X}}$ from its noise-free counterpart $\mathbf{X}=\mathbf{H S}_{K}$. To begin, it will be assumed that $M \geq K$ and that $\mathbf{H}$ is full rank, but these assumptions will be relaxed in Section IV, as will the restriction to the single-user case.

Let the $N \times 1$ vector $\mathbf{s}_{k}$ represent the symbol sequence transmitted from antenna $k$, and let $\mathbf{s}_{k, r}, \mathbf{s}_{k, i}$ represent its real and imaginary parts, respectively. Defining

$$
\tilde{\mathbf{s}}_{k}=\left[\begin{array}{c}
\mathbf{s}_{k, r} \\
\mathbf{s}_{k, i}
\end{array}\right],
$$


the signal matrix is decomposed as

$$
\left[\begin{array}{c}
\operatorname{real}\left(\mathbf{S}_{K}^{T}\right) \\
\operatorname{imag}\left(\mathbf{S}_{K}^{T}\right)
\end{array}\right]=\left[\begin{array}{ccc}
\mathbf{s}_{1, r} & \cdots & \mathbf{s}_{K, r} \\
\mathbf{s}_{1, i} & \cdots & \mathbf{s}_{K, i}
\end{array}\right]=\left[\begin{array}{lll}
\tilde{\mathbf{s}}_{1} & \cdots & \tilde{\mathbf{s}}_{K}
\end{array}\right] .
$$

The primary assumption of the algorithm presented in this paper is that the signals are encoded in the following manner:

$$
\tilde{\mathbf{s}}_{k}=\mathcal{Q}_{k} \tilde{\mathbf{s}}_{1}, \quad k=2, \cdots, K
$$

where $\mathcal{Q}_{k}$ is a full-rank, real-valued, $2 N \times 2 N$ transformation matrix. While some of the results presented in the paper will hold for a completely general $\mathcal{Q}_{k}$, others will require $\mathcal{Q}_{k}$ to have the following form:

$$
\mathcal{Q}_{k}=\left[\begin{array}{cc}
\operatorname{real}\left(\mathbf{Q}_{k}\right) & -\operatorname{imag}\left(\mathbf{Q}_{k}\right) \\
\operatorname{imag}\left(\mathbf{Q}_{k}\right) & \operatorname{real}\left(\mathbf{Q}_{k}\right)
\end{array}\right],
$$

so that

$$
\mathbf{s}_{k}=\mathbf{Q}_{k} \mathbf{s}_{1}, \quad k=2, \cdots, K
$$

for a given complex $\mathbf{Q}_{k}$.

Equations (2) and (4) provide a general framework that encompasses many types of popular space-time codes:

Example 1 - The $K=2$ STBC of [1] satisfies (2) with

$$
\begin{aligned}
& \mathcal{Q}_{1}=\left[\begin{array}{cc}
\mathbf{I}_{\frac{N}{2}} \otimes(\mathbf{J} \tilde{\mathbf{I}}) & 0 \\
0 & \mathbf{I}_{N}
\end{array}\right] \\
& \mathcal{Q}_{2}=\left[\begin{array}{cc}
\mathbf{I}_{\frac{N}{2}} \otimes \tilde{\mathbf{I}} & 0 \\
0 & \mathbf{I}_{\frac{N}{2}} \otimes \mathbf{J}
\end{array}\right],
\end{aligned}
$$

where $\mathbf{I}_{a}$ indicates an $a \times a$ identity matrix, and

$$
\tilde{\mathbf{I}}=\left[\begin{array}{ll}
0 & 1 \\
1 & 0
\end{array}\right] \quad \mathbf{J}=\left[\begin{array}{cc}
0 & 1 \\
-1 & 0
\end{array}\right]
$$

Similar transformations exist for the STBCs with larger values of $K$ described in [2], [3].

Example 2 - The code described in [13] combines the structure of the $K=2$ STBC together with the use of two "subprecoders" $\mathbf{C}_{1}$ and $\mathbf{C}_{2}$. When cast in the framework of (2), this method results in

$\mathcal{Q}_{1}=\left[\begin{array}{cccc}\operatorname{real}\left(\mathbf{C}_{1}\right) & 0 & -\operatorname{imag}\left(\mathbf{C}_{1}\right) & 0 \\ 0 & -\operatorname{real}\left(\mathbf{C}_{2}\right) & 0 & \operatorname{imag}\left(\mathbf{C}_{2}\right) \\ \operatorname{imag}\left(\mathbf{C}_{1}\right) & 0 & \operatorname{real}\left(\mathbf{C}_{1}\right) & 0 \\ 0 & \operatorname{imag}\left(\mathbf{C}_{2}\right) & 0 & \operatorname{real}\left(\mathbf{C}_{2}\right)\end{array}\right]$

$\mathcal{Q}_{2}=\left[\begin{array}{cc}\mathbf{J} \otimes \mathbf{I}_{\frac{N}{2}} & 0 \\ 0 & -\mathbf{J} \otimes \mathbf{I}_{\frac{N}{2}}\end{array}\right] \mathcal{Q}_{1}$

where $\mathbf{J}$ is defined in (5).

Example 3 - The so-called linear dispersion (LD) codes of [12] satisfy (2) as well. For these codes,

$$
\mathbf{S}^{T}=\sum_{q=1}^{N_{u}} \alpha_{q} \mathbf{A}_{q}+j \beta_{q} \mathbf{B}_{q}
$$

where $\alpha_{q}$ and $\beta_{q}$ are real scalars, and $\mathbf{A}_{q}, \mathbf{B}_{q}$ are $N \times K$ complex matrices. The LD approach is equivalent to (2) with the definitions

$$
\begin{aligned}
& \mathcal{Q}_{k}=\left[\begin{array}{rr}
\operatorname{real}\left(\mathcal{A}_{k}\right) & -\operatorname{imag}\left(\mathcal{B}_{k}\right) \\
\operatorname{imag}\left(\mathcal{A}_{k}\right) & \operatorname{real}\left(\mathcal{B}_{k}\right)
\end{array}\right] \\
& \mathcal{A}_{k}=\left[\begin{array}{lll}
\mathbf{A}_{1}(:, k) & \cdots & \mathbf{A}_{N_{u}}(:, k)
\end{array}\right] \\
& \mathcal{B}_{k}=\left[\begin{array}{lll}
\mathbf{B}_{1}(:, k) & \cdots & \mathbf{B}_{N_{u}}(:, k)
\end{array}\right]
\end{aligned}
$$

where $(:, k)$ denotes the $k^{\text {th }}$ column of the associated matrix.

Example 4 - For the code described in [11], $\mathbf{S}$ is circulant and Hankel, so (3) applies with

$$
\mathbf{Q}_{k}=\left[\begin{array}{cc}
0 & \mathbf{I}_{N-1} \\
1 & 0
\end{array}\right]^{k-1}
$$

Example 5 - For the modulation-induced code of [8], [9], $\mathbf{Q}_{k}$

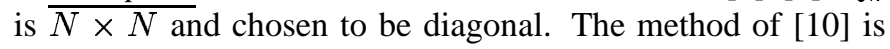
similar, except it mixes several independent symbol streams together with different coding matrices for each signal and each transmit antenna (i.e., $\mathbf{S}_{K}$ is a sum of the type of single-user signal matrices employed by [8], [9]).

As described in the next section, the structure induced by (2) and (4) leads to zero-forcing receivers for each transmit antenna that are related via linear transformations that can be determined directly from the data without knowledge of the channel. In certain cases, this allows construction of a data-derived matrix whose one-dimensional nullspace will, in the noiseless case, contain the weight vector that recovers $\mathbf{s}_{1}$.

\section{DIRECT LINEAR RECEIVER ESTIMATION}

Assume that the transmitted symbols and transformation matrices have been chosen so that $\mathbf{S}_{K}$ is full rank. To describe the proposed algorithm, consider an SVD of the rank $K$ noiseless matrix $\mathbf{X}$ :

$$
\mathbf{X}=\mathbf{H S}_{K}=\mathbf{U} \boldsymbol{\Sigma} \mathbf{V}^{*},
$$

where $\mathbf{U}$ is $M \times K, \boldsymbol{\Sigma}$ is $K \times K$, and $\mathbf{V}$ is $N \times K$. If $\mathbf{S}_{K}$ is full rank, there exists a full-rank $K \times K$ matrix $\mathbf{H}_{v}$ that satisfies

$$
\mathbf{V}^{*}=\mathbf{H}_{v} \mathbf{S}_{K} \quad \Longrightarrow \quad \overline{\mathbf{V}}=\mathbf{S}_{K}^{T} \mathbf{H}_{v}^{T}
$$

where $(\bar{\cdot})$ denotes conjugation. The goal of the method described below is to find a linear weight vector $\mathbf{w}_{1}$ such that

$$
\overline{\mathbf{V}} \mathbf{w}_{1}=\mathbf{s}_{1}
$$

without knowledge of $\mathbf{H}$ or, equivalently, $\mathbf{H}_{v}$. Rewriting this equation in terms of real and imaginary parts yields

$$
\mathcal{V} \tilde{\mathbf{w}}_{1}=\tilde{\mathbf{s}}_{1}
$$

where

$$
\mathcal{V}=\left[\begin{array}{cc}
\operatorname{real}(\mathbf{V}) & \operatorname{imag}(\mathbf{V}) \\
-\operatorname{imag}(\mathbf{V}) & \operatorname{real}(\mathbf{V})
\end{array}\right]
$$

and $\tilde{\mathbf{w}}_{1}$ is defined similarly to $\tilde{\mathbf{s}}_{1}$. 


\section{A. Blind Estimation}

Defining $\tilde{\mathbf{w}}_{k}$ to be the linear receiver weights that recover $\tilde{\mathbf{s}}_{k}$ from $\mathcal{V}$, it follows from (2) and (10) that

$$
\mathcal{V} \tilde{\mathbf{w}}_{k}=\tilde{\mathbf{s}}_{k}=\mathcal{Q}_{k} \tilde{\mathbf{s}}_{1}=\mathcal{Q}_{k} \mathcal{V} \tilde{\mathbf{w}}_{1}
$$

and hence that

$$
\tilde{\mathbf{w}}_{k}=\mathcal{V}^{T} \mathcal{Q}_{k} \mathcal{V} \tilde{\mathbf{w}}_{1}
$$

since $\mathcal{V}$ has orthonormal columns. Note that the linear transformation that relates the zero-forcing receivers with one another does not explicitly depend on the channel, only on known or data-derived quantities. Furthermore,

$$
\begin{aligned}
\tilde{\mathbf{s}}_{1} & =\mathcal{V} \tilde{\mathbf{w}}_{1} \\
& =\mathcal{Q}_{k}^{-1} \mathcal{V} \tilde{\mathbf{w}}_{k} \\
& =\mathcal{Q}_{k}^{-1} \mathcal{V} \mathcal{V}^{T} \mathcal{Q}_{k} \mathcal{V} \tilde{\mathbf{w}}_{1}
\end{aligned}
$$

and subtracting (15) from (13) leads to the following equation for $\tilde{\mathbf{w}}_{1}$ :

$$
\mathcal{Q}_{k}^{-1} \mathbf{P}_{\mathcal{V}}^{\perp} \mathcal{Q}_{k} \mathcal{V} \mathbf{w}_{1}=0
$$

where $\mathbf{P}_{\mathcal{V}}^{\perp}=\mathbf{I}-\mathcal{V} \mathcal{V}^{T}$. Combining all such equations together for $k=2, \cdots, K$ yields

$$
\left[\begin{array}{c}
\mathcal{Q}_{2}^{-1} \mathbf{P}_{\mathcal{V}}^{\perp} \mathcal{Q}_{2} \mathcal{V} \\
\vdots \\
\mathcal{Q}_{K}^{-1} \mathbf{P}_{\mathcal{V}}^{\perp} \mathcal{Q}_{K} \mathcal{V}
\end{array}\right] \tilde{\mathbf{w}}_{1} \stackrel{\text { def }}{=} \tilde{\mathbf{Z}} \tilde{\mathbf{w}}_{1}=0
$$

If (4) holds, then (12) and (17) become

$$
\begin{gathered}
\mathbf{w}_{k}=\mathbf{V}^{T} \mathbf{Q}_{k} \overline{\mathbf{V}} \mathbf{w}_{1} \\
{\left[\begin{array}{c}
\mathbf{Q}_{2}^{-1} \mathbf{P}_{\overline{\mathbf{V}}}^{\perp} \mathbf{Q}_{2} \overline{\mathbf{V}} \\
\vdots \\
\mathbf{Q}_{K}^{-1} \mathbf{P}_{\overline{\mathbf{V}}}^{\perp} \mathbf{Q}_{K} \overline{\mathbf{V}}
\end{array}\right] \mathbf{w}_{1} \stackrel{\text { def }}{=} \mathbf{Z} \mathbf{w}_{1}=0}
\end{gathered}
$$

If $\tilde{\mathbf{Z}}$ (or $\mathbf{Z}$ ) has a nullspace of dimension one, then $\tilde{\mathbf{w}}_{1}$ (or $\mathbf{w}_{1}$ ) and hence $\mathbf{s}_{1}$ can be found from (17), to within the scalar ambiguity common to all blind equalizers. General conditions under which $\tilde{\mathbf{Z}}$ and $\mathbf{Z}$ have a rank-one nullspace are currently under investigation, but it has been observed via simulation that this property is generically true for the codes in [8], [9], [11] described above. One important situation where the property does not hold is for the STBC schemes of [1], [2], [3]. This is due to a fundamental ambiguity associated with blind decoding of STBCs, namely that $\mathbf{A} \mathbf{S}_{2}$ has exactly the same structure as $\mathbf{S}_{2}$ for any $\mathbf{A}$ of the form

$$
\mathbf{A}=\left[\begin{array}{rr}
a_{1} & -\bar{a}_{2} \\
a_{2} & \bar{a}_{1}
\end{array}\right] .
$$

Thus, it is impossible to distinguish between $\mathbf{H S}_{2}$ and $\mathbf{H A}{ }^{-1} \mathbf{A S}_{2}$ using only the STBC structure. The ambiguity can be resolved by the insertion of pilot symbols in the data, as was done in [4]. Another approach of course is to appropriately modify the structure of the space-time code, as done for example in [5], where a different linear precoder is used for even and odd symbols. The resulting modification of $\mathcal{Q}_{2}$ would then lead to a non-degenerate $\tilde{\mathbf{Z}}$ with the desired nullspace dimension. Based on the above discussion, it is clear that the transformation matrices should not be chosen so that $\mathcal{Q}_{k}^{-1}=\alpha \mathcal{Q}_{k}$ for some scalar $\alpha$. However, additional work is needed to determine other more general conditions that guarantee a unique solution.

In the presence of noise, (17) will of course not hold exactly. A reasonable approach in this case is to estimate $\tilde{\mathbf{w}}_{1}$ as the right singular vector of $\tilde{\mathbf{Z}}$ with smallest singular value, where $\mathcal{V}$ is replaced by an estimate obtained from the $K$ dominant right singular vectors of $\hat{\mathbf{X}}$. A similar argument holds for the case of (18). This is the method used in the simulations of Section VI.

\section{B. Semi-Blind Estimation}

One advantage of the method described above is that it is a simple matter to incorporate knowledge of any embedded training symbols into the estimate to improve performance. Suppose that $L$ elements of $\mathbf{s}_{1}$ are known, and let $\mathcal{I}$ denote the $2 L$-element set of indices corresponding to the locations of the known values in $\tilde{\mathbf{s}}_{1}$. Then the vector $\tilde{\mathbf{u}}=\tilde{\mathbf{s}}_{1}(\mathcal{I})$ is known, and a linear receiver that satisfies

$$
\mathcal{V}(\mathcal{I},:) \tilde{\mathbf{w}}_{1}=\tilde{\mathbf{u}}
$$

is desired, where $\mathcal{V}(\mathcal{I},:)$ is the matrix formed from the rows of $\mathcal{V}$ corresponding to $\mathcal{I}$ (as in Matlab notation). The blind solution of (17) is then augmented with (19) as follows:

$$
\left[\begin{array}{c}
\tilde{\mathbf{Z}} \\
\mathcal{V}(\mathcal{I},:)
\end{array}\right] \tilde{\mathbf{w}}_{1}=\left[\begin{array}{c}
0 \\
\tilde{\mathbf{u}}
\end{array}\right],
$$

and the semi-blind estimate is obtained as

$$
\hat{\tilde{\mathbf{w}}}_{1}=\left[\begin{array}{c}
\tilde{\mathbf{Z}} \\
\mathcal{V}(\mathcal{I},:)
\end{array}\right]^{\dagger}\left[\begin{array}{c}
0 \\
\tilde{\mathbf{u}}
\end{array}\right],
$$

where $(\cdot)^{\dagger}$ denotes a pseudo-inverse. The presence of training symbols removes the ambiguity associated with the blind estimate of $\tilde{\mathbf{w}}_{1}$. An identical approach can be used when (18) holds.

\section{EXTENSIONS OF THE Algorithm}

\section{A. Multiple Users}

If $d$ (symbol synchronous) users are present, then equation (1) becomes

$$
\hat{\mathbf{X}}=\mathcal{H} \mathcal{S}+\mathbf{N}
$$

where

$$
\mathcal{H}=\left[\begin{array}{lll}
\mathbf{H}_{1} & \cdots & \mathbf{H}_{d}
\end{array}\right] \quad M \times K
$$




$$
\begin{aligned}
\mathcal{S} & =\left[\begin{array}{c}
\mathbf{S}_{K_{1}, 1} \\
\vdots \\
\mathbf{S}_{K_{d}, d}
\end{array}\right] \quad K \times N \\
K & =\sum_{i=1}^{d} K_{i} .
\end{aligned}
$$

and where $\mathbf{S}_{K_{i}, i}, \mathbf{H}_{i}, K_{i}$ represent respectively the transmitted signals, channel, and number of transmit antennas for the $i^{\text {th }}$ user. Assume that each user employs space-time coding in the form of (2), with $\mathcal{Q}_{k, i}$ representing the $k^{\text {th }}$ transformation for user $i$. Note that data obeying (22) could also be generated by a single user whose transmit antennas are divided into $d$ groups, with each group transmitting a different data sequence. Such an approach could be used to trade off diversity for throughput.

When $M \geq K$, two separate situations must be considered (if $M<K$, methods similar to those described below in Section IV-B below must be used):

Case 1-All users employ different space-time codes. In this situation, no modification of the blind or semi-blind algorithm described above is required, provided that each user's code is "different enough." In the blind case, the linear receiver for user $i$ is found from the following equation

$$
\left[\begin{array}{c}
\mathcal{Q}_{2, i}^{-1} \mathbf{P}_{\mathcal{V}}^{\perp} \mathcal{Q}_{2, i} \mathcal{V} \\
\vdots \\
\mathcal{Q}_{K_{i}, i}^{-1} \mathbf{P}_{\mathcal{V}}^{\perp} \mathcal{Q}_{K_{i}, i} \mathcal{V}
\end{array}\right] \tilde{\mathbf{w}}_{1, i} \stackrel{\text { def }}{=} \tilde{\mathbf{Z}}_{i} \tilde{\mathbf{w}}_{1, i}=0
$$

If all of the users' codes have been appropriately chosen, then $\tilde{\mathbf{Z}}_{i}$ will have a rank-one nullspace and the solution is unique (to within a scalar).

Case 2 - All users employ the same space-time code. Only one $\tilde{\mathbf{Z}}$ matrix may be formed in this case, and it will have a nullspace of dimension $d$ that is spanned by $\left[\begin{array}{ccc}\tilde{\mathbf{w}}_{1,1} & \cdots & \tilde{\mathbf{w}}_{1, d}\end{array}\right]$. The nullspace basis could be used to reduce the dimension of the data to $d$, but additional information would be required to separate the individual user's symbol sequences in a second step, using for example the assumption of constant modulus [14] or finite alphabet signals [15]. An alternative approach is presented in Section V, where it is shown how to exploit space-time codes and the constant modulus assumption simultaneously in a single step algorithm. One exception to the approach outlined for Case 2 occurs when one of the users has more transmit antennas than the others. This user's weight vector can be found using (23), since it will be the only one that satisfies (23) for that particular value of $K_{i}$.

If each user transmits linearly independent training data, then the semi-blind approach of Section III-B can be used directly for either of the two cases described above. If $\mathcal{I}_{i}$ and $\mathbf{u}_{i}$ represent the time indices and data associated with the training data, then $\tilde{\mathbf{w}}_{1, i}$ may be found using

$$
\hat{\tilde{\mathbf{w}}}_{1, i}=\left[\begin{array}{c}
\tilde{\mathbf{Z}}_{i} \\
\mathcal{V}\left(\mathcal{I}_{i},:\right)
\end{array}\right]^{\dagger}\left[\begin{array}{c}
0 \\
\tilde{\mathbf{u}}_{i}
\end{array}\right]
$$

As before, similar equations can be found for the case where (18) holds.

\section{B. More Transmit Than Receive Antennas}

A simple modification of the above algorithm allows spacetime codes of the form in (3) to work in situations where $M<$ $K$. Assume that $\mathbf{Q}_{k}=\mathbf{Q}^{k-1}$ for $k=2, \cdots, K$ and some $\mathbf{Q}$ (always true for $K=2$ ). This constraint is satisfied by the circulant code of [11] and can be implemented with the coding approach of [8], [9]. Under these assumptions, $\mathbf{s}_{k}=\mathbf{Q}^{k-1} \mathbf{s}_{1}$, and

$$
\begin{aligned}
\mathbf{X Q}^{T} & =\mathbf{H S}_{K} \mathbf{Q}^{T} \\
& =\left[\begin{array}{ll}
\mathbf{0} & \mathbf{H}
\end{array}\right]\left[\begin{array}{c}
\mathbf{S}_{K} \\
\mathbf{s}_{1}^{T}\left(\mathbf{Q}^{T}\right)^{K}
\end{array}\right] \\
& =\left[\begin{array}{ll}
\mathbf{0} & \mathbf{H}
\end{array}\right] \mathbf{S}_{K+1},
\end{aligned}
$$

where $\mathbf{0}$ is a $M \times 1$ vector of zeros. Since

$$
\mathbf{H S}_{K}=\left[\begin{array}{ll}
\mathbf{H} & 0
\end{array}\right] \mathbf{S}_{K+1},
$$

a stacking operation leads to

$$
\left[\begin{array}{c}
\mathbf{X} \\
\mathbf{X Q}^{T}
\end{array}\right]=\mathcal{H} \mathbf{S}_{K+1}
$$

where $\mathcal{H}$ is a $2 M \times(K+1)$ block Sylvester matrix identical in form to those obtained in blind equalization problems [16]. Thus, the same data model is obtained as before, except the number of effective receive antennas has been doubled, while the number of effective transmit antennas has increased by only one. Stacking $P-1$ times and adding the effects of noise leads to

$$
\hat{\mathcal{X}} \stackrel{\text { def }}{=}\left[\begin{array}{l}
\hat{\mathbf{X}} \\
\hat{\mathbf{X}} \mathbf{Q}^{T} \\
\vdots \\
\hat{\mathbf{X}}\left(\mathbf{Q}^{T}\right)^{P-1}
\end{array}\right]=\mathcal{H} \mathbf{S}_{K+P-1}+\mathcal{N}
$$

where $\mathcal{N}$ is formed identically to $\hat{\mathcal{X}}$. The new "channel" matrix $\mathcal{H}$ is guaranteed to be full rank as long as $\mathbf{H}$ is, and will have at least as many rows as columns provided that

$$
P \geq \frac{K-1}{M-1} \text {. }
$$

The algorithms of Section III can then be directly applied to $\hat{\mathcal{X}}$ rather than $\hat{\mathbf{X}}$. Note that, in general, the stacking operation will lead to a noise term $\mathcal{N}$ that is neither temporally or spatially white, even if $\mathbf{N}$ was. This can be easily accounted for, however, by prewhitening in both space and time.

\section{Rank Deficient Channels}

If the channel is rank deficient, i.e.,

$$
\operatorname{rank}(\mathbf{H})=\rho<\min \{M, K\},
$$


then in the noiseless case, $\operatorname{rank}(\mathbf{X})=\rho$. If the SVD of $\mathbf{X}$ in (7) is partitioned so that $\mathbf{V}$ contains the first $\rho$ right singular vectors, then (8) still holds except that $\mathbf{H}_{v}$ will be $\rho \times K$. The transpose of (8) will then be equivalent to a noiseless version of the original model (1) for a case with more transmit than receive antennas (i.e., $\mathbf{H}_{v}$ is fat), and the approach of Section IV-B can be used. Instead of (29), the algorithms are applied to the matrix

$$
\mathcal{X} \stackrel{\text { def }}{=}\left[\begin{array}{l}
\mathbf{V}^{*} \\
\mathbf{V}^{*} \mathbf{Q}^{T} \\
\vdots \\
\mathbf{V}^{*}\left(\mathbf{Q}^{T}\right)^{P-1}
\end{array}\right],
$$

where $P$ is chosen to satisfy

$$
P \geq \frac{K-1}{\rho-1} .
$$

\section{Exploiting Constant Modulus Signals}

The Analytic Constant Modulus (ACM) algorithm of [14] is a technique for blind source separation that exploits the constant modulus (CM) property to directly estimate linear receivers for all CM signals present. Though computationally complex, the ACM algorithm has been shown to provide excellent performance with very short data records. If applied to data generated by $d$ users employing space-time coding with $K_{1}, \cdots, K_{d}$ transmit antennas, respectively, then the ACM approach would estimate linear receivers for $K=\sum K_{i}>d$ signals (provided that $M>K$ ) even though the signals from a given user are not independent. The approach presented here uses the relationship of (18) between the weight vectors to reduce the number of solutions to at most $d$, depending on whether or not the users employ different codes.

In the absence of noise, the CM property is enforced by finding linear receivers that satisfy

$$
\mathbf{w}^{*} \mathbf{v}_{n} \mathbf{v}_{n}^{*} \mathbf{w}=\left(\overline{\mathbf{v}}_{n} \otimes \mathbf{v}_{n}\right)^{*}(\overline{\mathbf{w}} \otimes \mathbf{w})=1 \quad n=1, \cdots, N
$$

where $\mathbf{v}_{n}^{*}$ is the $n^{\text {th }}$ row of $\overline{\mathbf{V}}$ in (7), and where, without loss of generality, unity modulus is assumed. Stacking all $N$ such equations together yields

$$
\left[\begin{array}{c}
\left(\overline{\mathbf{v}}_{1} \otimes \mathbf{v}_{1}\right)^{*} \\
\vdots \\
\left(\overline{\mathbf{v}}_{N} \otimes \mathbf{v}_{N}\right)^{*}
\end{array}\right](\overline{\mathbf{w}} \otimes \mathbf{w}) \stackrel{\text { def }}{=} \mathbf{P}_{1}(\overline{\mathbf{w}} \otimes \mathbf{w})=\mathbf{1},
$$

where $\mathbf{1}$ is a vector of ones. The ACM algorithm finds the nullspace of $\left[\begin{array}{ll}\mathbf{P}_{1} & -\mathbf{1}\end{array}\right]$ and then uses a joint diagonalization step to find basis vectors of the form $\overline{\mathbf{w}} \otimes \mathbf{w}$ to separate the users. For the problem considered in this paper, if $M>K$ there would be $K$ solutions, $K_{i}$ for each user.

To show how the structure induced by space-time coding can be used to eliminate "redundant" solutions, suppose there are $1 \leq d^{\prime} \leq d$ users each with $K^{\prime}$ transmit antennas that employ the same space-time code $\left\{\mathbf{Q}_{2}, \cdots, \mathbf{Q}_{K^{\prime}}\right\}$. Because of (18), not only will $\mathbf{w}_{1, i}$ satisfy (31) for $i=1, \cdots, d^{\prime}$, so will $\mathbf{V}^{T} \mathbf{Q}_{k} \overline{\mathbf{V}} \mathbf{w}_{1, i}$, and hence

$$
\begin{gathered}
{\left[\begin{array}{c}
\left(\mathbf{V}^{*} \mathbf{Q}_{k}^{T} \mathbf{V} \overline{\mathbf{v}}_{1} \otimes \mathbf{V}^{T} \mathbf{Q}_{k}^{*} \overline{\mathbf{V}} \mathbf{v}_{1}\right)^{*} \\
\vdots \\
\left(\mathbf{V}^{*} \mathbf{Q}_{k}^{T} \mathbf{V} \overline{\mathbf{v}}_{N} \otimes \mathbf{V}^{T} \mathbf{Q}_{k}^{*} \overline{\mathbf{V}} \mathbf{v}_{N}\right)^{*}
\end{array}\right]\left(\overline{\mathbf{w}}_{1, i} \otimes \mathbf{w}_{1, i}\right)} \\
\stackrel{\text { def }}{=} \mathbf{P}_{k}\left(\overline{\mathbf{w}}_{1, i} \otimes \mathbf{w}_{1, i}\right)=\mathbf{1}
\end{gathered}
$$

for $i=1, \cdots, d^{\prime}$ and $k=2, \cdots, K^{\prime}$. Stacking all $K^{\prime}$ equations together yields

$$
\left[\begin{array}{c}
\mathbf{P}_{1} \\
\vdots \\
\mathbf{P}_{K^{\prime}}
\end{array}\right]\left(\overline{\mathbf{w}}_{1, i} \otimes \mathbf{w}_{1, i}\right) \stackrel{\text { def }}{=} \mathbf{P}\left(\overline{\mathbf{w}}_{1, i} \otimes \mathbf{w}_{1, i}\right)=\left[\begin{array}{c}
\mathbf{1} \\
\vdots \\
\mathbf{1}
\end{array}\right]
$$

which will generically have only $d^{\prime}$ solutions, one for each user, provided the space-time code is properly designed. As with the ACM algorithm, the individual users' solutions are found by applying the joint diagonalization procedure to the nullspace basis vectors of $\left[\begin{array}{ll}\mathbf{P}-\mathbf{1}\end{array}\right]$. A dimension reduction step (as described in [17]) is required, however, since the $d^{\prime}$ different $K \times K$ matrices to be jointly diagonalized will each have rank $d^{\prime}$. The algorithm outlined above can be repeated for each set of users employing the same space-time code.

If $d^{\prime}=1$, the nullspace of $\left[\begin{array}{ll}\mathbf{P}-\mathbf{1}\end{array}\right]$ has dimension one, and is spanned by a single vector. If $\mathbf{y}$ represents this vector, the desired linear receiver is found by simply performing a rankone factorization of $\mathbf{Y}=\operatorname{vec}^{-1}(\mathbf{y})$, where $\operatorname{vec}^{-1}(\mathbf{y})$ refers to the procedure of forming a $K \times K$ matrix from the $K^{2} \times 1$ vector $\mathbf{y}$.

\section{Simulation EXAmples}

The first example considers a case with one user, $M=3$ receive antennas, $K=2$ transmit antennas, and a $N=50$ sample block of data. Three different space-time coding strategies were implemented: (1) the circulant code of [11], (2) the code of [8], [9] with a diagonal $\mathbf{Q}_{2}$ whose entries were drawn at random from the unit circle, and (3) a random unitary $\mathbf{Q}_{2}$. Unit-amplitude QPSK symbols were generated for the transmitted signals, and the elements of the channel and noise matrices were zero-mean, circular complex Gaussian random variables, with variances chosen to achieve the desired SNR. In the plots shown, the SNR is defined by $\sigma_{h}^{2} / \sigma_{n}^{2}$, where $\sigma_{h}^{2}$ and $\sigma_{n}^{2}$ are the variances of the elements of $\mathbf{H}$ and $\mathbf{N}$ respectively. A new ran$\operatorname{dom} \mathbf{H}, \mathbf{N}$, and $\mathbf{s}_{1}$ are generated for each trial, and used for each of the three coding algorithms one-at-a-time. A variable number $L$ of known symbols were assumed to be present, and were used in (21) to estimate the linear receiver for each algorithm. The symbol error rate was then calculated from the resulting estimate of $\hat{\mathbf{s}}_{1}$. Figure 1 shows the results of the algorithms as a function of $L$ for three different SNRs. Note that all three codes have nearly identical performance. 


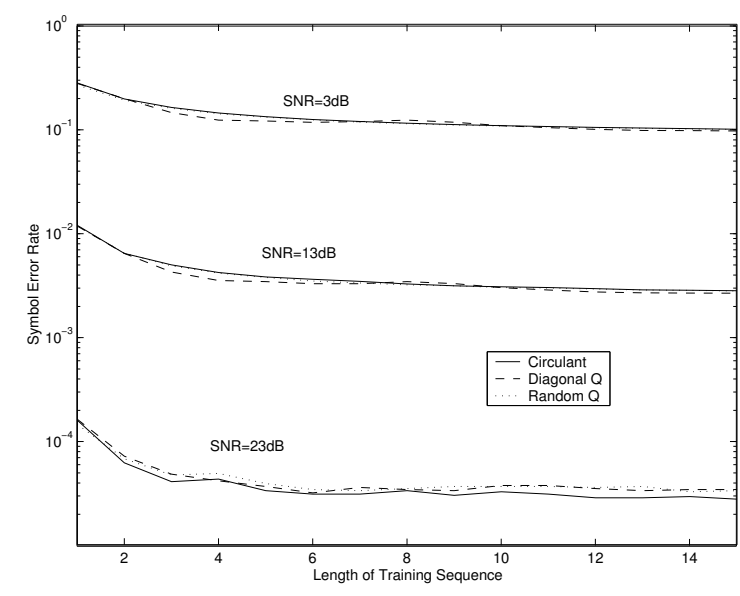

Fig. 1. Semi-Blind Symbol Error Rate versus Training Interval $L$ for 3 SpaceTime Coding Schemes.

The second example is similar to the first, except only the circulant code is implemented, and the ACM approach of Section $\mathrm{V}$ is used. Only one training symbol $(L=1)$ is assumed to be known, and it is only used after the signal estimate is obtained in order to eliminate the scalar ambiguity of the blind estimate. Figure 2 shows the resulting symbol error rate as a function of $N$ for three different SNRs. Compared with Figure 1, there is a dramatic reduction in error rate that results from exploiting the constant modulus property, even for very small values of $N$.

\section{CONCLUSIONS}

Two new techniques have been presented for direct, closedform, blind and semi-blind estimation of linear receivers for certain types of space-time codes. Applicable codes fall in a general framework that includes as special cases several previously proposed space-time transmission schemes. Simulations show excellent performance with very small block sizes, especially when the constant modulus property can be exploited.

\section{ACKNOWLEDGEMENT}

This work was supported by the National Science Foundation under Wireless Initiative Grant CCR-9979452 and Information Technology Research Grant CCR-0081476.

\section{REFERENCES}

[1] S. Alamouti, "A Simple Transmit Diversity Technique for Wireless Communications", IEEE J. Select. Areas Commun., 16(8):1451-1458, August 1998.

[2] V. Tarokh, H. Jafarkhani, and A. R. Calderbank, "Space-Time Block Codes from Orthogonal Designs", IEEE Trans. Info. Theory, 45(5):14561467, 1999.

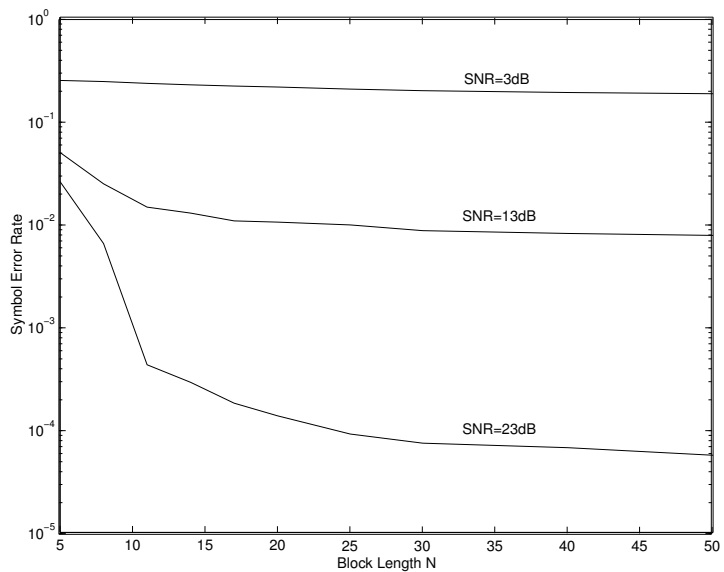

Fig. 2. Symbol Error Rate versus Block Length $N$ for the Circulant Code using the ACM Algorithm.

[3] G. Ganesan and P. Stoica, "Space-Time Diversity", In Signal Processing Advances in Wireless and Mobile Communications, G. Giannakis, Y. Hua, P. Stoica, and L. Tong, editors, volume 2, pages 59-88, Chapter 2. Prentice-Hall, Inc., 2001.

[4] Z. Liu, G. Giannakis, A. Scaglione, and S. Barbarossa, "Decoding and Equalization of Unknown Multipath Channels Based on Block Precoding and Transmit-Antenna Diversity", In Proc. 33rd Asilomar Conf. on Signals, Systems, and Computers, volume 2, pages 1557-1561, 1999.

[5] Z. Liu, G. Giannakis, B. Muquet, and S. Zhou, "Space-Time Coding for Broadband Wireless Communications", Wireless Communications and Mobile Computing, 1(1):35-53, 2001.

[6] C. Budianu and L. Tong, "Channel Estimation for Space-Time Orthogonal Block Codes”, In Proc. Int'l Conf. on Communications, Helsinki, Finland, June 2001.

[7] P. Stoica and G. Ganesan, "Space-Time Block Codes: Trained, Blind and Semi-Blind Detection", submitted to IEEE Trans. on Sig. Proc., 2001.

[8] P. Vandaele, G. Leus, and M. Moonen, "A Non-Iterative Blind Signal Separation Algorithm Based on Transmit Diversity and Coding", In Proc. 33rd Asilomar Conf. Signals, Systems, and Computers, Oct. 1999.

[9] G. Leus, P. Vandaele, and M. Moonen, "Deterministic Blind ModulationInduced Source Separation for Digital Wireless Communications", IEEE Trans. Sig. Proc., 49(1):219-227, Jan. 2001.

[10] H. Artés and F. Hlawatsch, "Blind Equalization of MIMO Channels using Deterministic Precoding”, In Proc. ICASSP, volume VI, pages 21532156, Salt Lake City, UT, 2001.

[11] A. Swindlehurst, "Simultaneous Channel Estimation and Decoding for Diagonal Space-Time Codes", In Proc. Sensor Array \& Multichannel Proc. Workshop, pages 173-177, Cambridge, MA, 2000.

[12] B. Hassibi and B. Hochwald, "High-Rate Linear Space-Time Codes", In Proc. ICASSP, volume IV, pages 2461-2464, Salt Lake City, UT, 2001.

[13] Z. Liu, G. Giannakis, S. Barbarossa, and A. Scaglione, "TransmitAntenna Space-Time Block Coding for Generalized OFDM in the Presence of Unknown Multipath", IEEE J. Select. Areas Commun., 19(7):1352-1364, July 2001.

[14] A. van der Veen and A. Paulraj, "An Analytical Constant Modulus Algorithm”, IEEE Trans. Sig. Proc., 44(5):1136-1157, May 1996.

[15] S. Talwar, M. Viberg, and A. Paulraj, "Blind Separation of Synchronous Co-Channel Digital Signals Using an Antenna Array. Part I. Algorithms", IEEE Trans. Sig. Proc., 44(5):1184-1197, May 1996.

[16] E. Moulines, P. Duhamel, J.F. Cardoso, and S. Mayrargue, "Subspace Methods for the Blind Identification of Multichannel FIR Filters", IEEE Trans. Sig. Proc., 43(2):516-525, Feb. 1995.

[17] A. van der Veen, "Joint Diagonalization via Subspace Fitting Techniques", In Proc. ICASSP, volume V, pages 2773-2776, Salt Lake City, UT, 2001. 